\title{
A COUPLED SCHEMA OF PROBABILISTIC ATLAS AND STATISTICAL SHAPE AND APPEARANCE MODEL FOR 3D PROSTATE SEGMENTATION IN MR IMAGES
}

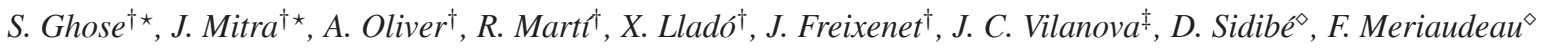 \\ ${ }^{\dagger}$ University of Girona, Computer Vision and Robotics Group, Girona, Spain. \\ $\ddagger$ Girona Magnetic Resonance Center, Girona, Spain. \\ $\diamond$ Université de Bourgogne, Le2i-UMR CNRS 6306, Le Creusot, France. \\ (soumya.ghose/jhimli.mitra/dro-desire.sidibe/fabrice.meriaudeau)@u-bourgogne.fr, (aoliver/marly/llado)eia.udg.edu
}

\begin{abstract}
A hybrid framework of probabilistic atlas and statistical shape and appearance model (SSAM) is proposed to achieve 3D prostate segmentation. An initial 3D segmentation of the prostate is obtained by registering the probabilistic atlas to the test dataset with deformable Demons registration. The initial results obtained are used to initialize multiple SSAMs corresponding to the apex, central and base regions of the prostate gland to incorporate local variabilities. Multiple mean parametric models of shape and appearance are derived from principal component analysis of prior shape and intensity information of the prostate from the training data. The parameters are then modified with the prior knowledge of the optimization space to achieve 2D segmentation. The 2D labels are registered to the 3D labels generated using probabilistic atlas to constrain the pose variation and generate valid $3 \mathrm{D}$ shapes. The proposed method achieves a mean Dice similarity coefficient value of $0.89 \pm 0.11$ and mean Hausdorff distance of $3.05 \pm 2.25 \mathrm{~mm}$ when validated with 15 prostate volumes of a public dataset in a leave-one-out validation framework.
\end{abstract}

Index Terms - Prostate segmentation, probabilistic atlas, statistical shape and appearance model.

\section{Introduction}

Prostate cancer is the most commonly diagnosed cancer in North America and accounted for 33,000 estimated deaths in 2011 [1]. Accurate prostate segmentation in magnetic resonance (MR) images aids in volume estimation, surgical planing and multi-modal image registration. However, manual segmentation of the prostate in MR images is time consuming and suffers from inter and intra observer differences.

Semi-automatic or automatic accurate prostate segmentation is a challenging task in MR images due to inter patient shape, size and intensity variabilities. In the last decade, atlas

Research funded by VALTEC 08-1-0039 of Generalitat de Catalunya, Spanish Sc. \& Innov. grant nb. TIN2011-23704, Spain \& Conseil Régional de Bourgogne, France. based prostate segmentation methods have produced accurate results $[2,3,4]$. Often a hybrid schema of atlas and shape constrained deformable models are used [3] to segment the prostate. Statistical shape models (SSM) [5] produce accurate prostate segmentation 3D. Zhu et al. [6] hybrid approach of locally optimized 2D segmentation and 3D registration to constrain the shape has provided accurate results. In recent years, Li et al.[7] adopted a similar hybrid 2D/3D approach to segment prostate in $3 \mathrm{D}$ CT images that produced good segmentation accuracies.

Motivated by [3] we adopt a similar probabilistic atlas and deformable model (SSAM) approach. We propose a novel hybrid framework in which a probabilistic prostate atlas is registered to the test dataset to obtain an probabilistic 3D segmentation and the segmentation achieved is used to initialize 2D multiple SSAMs corresponding to the apex, central and the base regions of the prostate to improve on local segmentation accuracies. Additionally, following the hybrid 2D/3D approach of [6], the optimal 2D segmentations using SSAMs are constrained by registering them to the 3D labels obtained from the probabilistic atlas. Such a process ensures that $2 \mathrm{D}$ boundaries, which are locally optimized on 2D slices can build a valid 3D shape with better segmentation accuracies compared to some of the works in literature $[8,9,10]$ that use the same prostate public dataset [11]. The key contributions of this work are,

1) The use of a hybrid framework of probabilistic atlas and statistical shape and appearance model to achieve segmentation.

2) The use of multiple statistical shape and appearance models corresponding to the base, central and the apex regions of the prostate to improve on the accuracies.

\section{Our Methodology}

The proposed method is developed on three major components: the probabilistic atlas based segmentation, the use of multiple SSAMs corresponding to the base, central and the apex regions of the prostate to improve on the $2 \mathrm{D}$ local seg- 


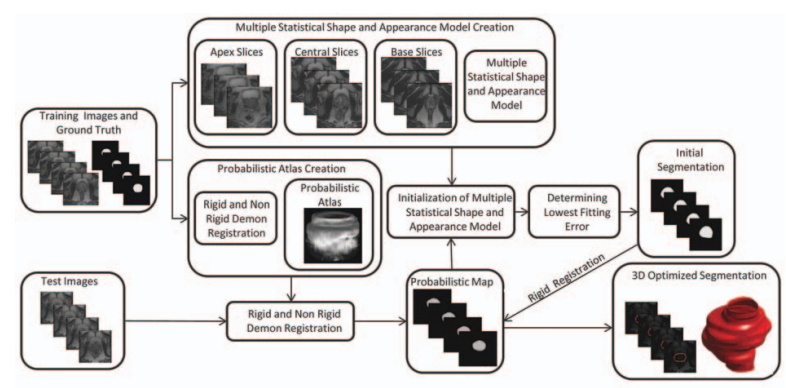

Fig. 1. Schematic representation of our approach. mentation accuracies and finally constraining the 2D segmentation by registering to the $3 \mathrm{D}$ segmentation achieved with probabilistic atlas. The schema of our proposed method is illustrated in Fig. 1.

\subsection{Probabilistic Atlas}

In recent years, Martin et al. [3] and Dowling et al. [9] have used Demons registration [12] to build atlases. Demons registration computes the pixel velocities or transformation field between the moving and reference images. The algorithm approaches the registration problem as a diffusion process. The displacement field is computed on a regular grid with one displacement vector per voxel. A set of forces, Demons are computed from the difference of pixel intensities at each node of the grid that drive the registration process. Edge forces of both the moving and reference images improve the registration convergence and stability while a normalization factor $\alpha$ adjusts the force strength. If $M$ and $F$ represent the moving and reference images respectively, then the pixel velocity $u$ at pixel $p$ with $m$ and $f$ as the respective pixel intensities is given by

$$
\mathbf{u}=\frac{(m-f) \nabla f}{|\nabla f|^{2}+\alpha(m-f)^{2}}+\frac{(m-f) \nabla m}{|\nabla m|^{2}+\alpha(m-f)^{2}}
$$

where $\nabla f$ and $\nabla m$ are the respective image gradients.

The process of atlas construction begins with alignment of $N$ manually segmented dataset to a common reference. One among $N$ datasets is manually selected by an expert to reduce bias and $N-1$ datasets are registered to the reference dataset. The registration is done in two stages, intensity based affine registration of $N-1$ datasets to the reference dataset is followed by the non-rigid Demons registration. The mean image is computed by averaging all patient images aligned to the reference image. Creation of the atlas is shown in Fig. 2. The probability map is obtained by averaging deformed patient label images. Given a new patient dataset, the dataset is first registered to the mean image using affine and Demons based registration. Once registered, inverse registration of the atlas probability map is used to determine the probabilistic segmentation of the new patient dataset. Motivated by the use of a spatially constrained deformable model to refine the results achieved with atlas based segmentation of Martin et al. [3] we propose to use the soft segmentation label to initialize multiple 2D SSAMs corresponding to the base, central and

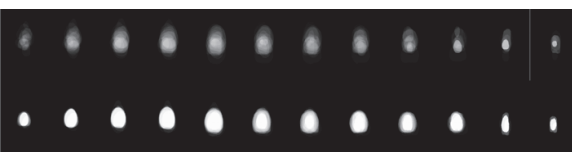

(a)

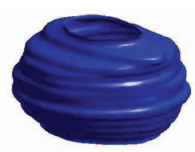

(b)
Fig. 2. (a) First row shows overlap of slices before registration and the second row shows overlap of slices after registration to a common reference. (b) The prostate atlas created from the registered slices.

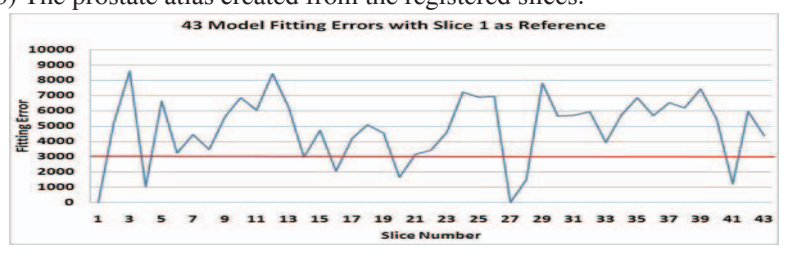

(a)

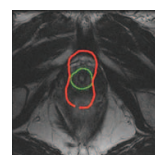

(b)

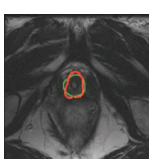

(c)

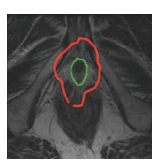

(d)

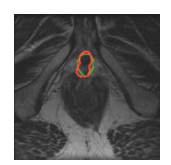

(e)
Fig. 3. (a) Mean models fitting errors for with dataset 1 as reference. (b),(d), segmentation without multiple mean model, (c),(e), segmentation with multiple mean model. The green contour gives the ground truth and the red contour gives the obtained result of some of the base slices.

the apex regions of the prostate to achieve optimized segmentation in each slice.

\subsection{Statistical shape and appearance model}

The objective of multiple 2D SSAM or active appearance model (AAM) [13] is to improve local 2D slice segmentation accuracies for the apex, central and the base regions of the prostate. Prostate segmentation is a challenging task in the base and the apex slices due to low contrast of the prostate in the images. However, SSAMs being region based techniques are more robust compared to edge based techniques like SSMs for the base and apex slices. Therefore, we build separate models for the apex, central and the base regions that incorporate region-based and shape-based information for each of these regions to learn the local variabilities. Traditional AAM is presented first, followed by a comprehensive discussion about multiple AAM to segment the prostate.

In traditional AAM, PCA of the point distribution models [13] of the manually segmented contours aligned to a common reference frame by generalized Procrustes analysis, is used to identify the principal modes of shape variations. PCA of intensity distributions warped into correspondence using a piece-wise affine warp and sampled from shape free reference, is used to identify the principal components of intensity variations. The shape and the intensity model may be formalized in the following manner. Let $E\{s\}$ and $E\{t\}$ represent the shape and intensity models of AAM where $s$ and $t$ are the shape and intensities of the corresponding training images, $\bar{s}$ and $\bar{t}$ are the mean shape and mean intensity, $\phi_{s}$ and $\phi_{t}$ are the truncated eigenvector matrices of shape and intensity respectively (obtained from $98 \%$ of the total variations), and 
$\theta_{s}$ and $\theta_{t}$ are the corresponding deformation parameters.

$$
E\{s\}=\bar{s}+\phi_{s} \theta_{s}, \quad E\{t\}=\bar{t}+\phi_{t} \theta_{t}
$$

The shape and intensity model are combined in a linear framework to give the combined model $b$ as,

$$
b=\left[\begin{array}{c}
W \theta_{s} \\
\theta_{t}
\end{array}\right]=\left[\begin{array}{c}
W \phi_{s}^{T}(E\{s\}-\bar{s}) \\
\phi_{t}^{T}(E\{t\}-\bar{t})
\end{array}\right]
$$

where $W$ denotes a weight factor (determined as in AAM [13]) coupling the intensity and the shape space. A third PCA of the combined model removes redundancy in the combined model giving $\widehat{b}$ as,

$$
\widehat{b}=V c
$$

where $V$ is the matrix of eigenvectors and $c$ the appearance parameters. Given a test image, the sum of squared difference of the intensities between the test image and mean model is minimized with respect to the pose (translation, rotation and scaling) parameters. Prior knowledge of the optimization space is acquired by perturbing the combined model and the pose parameter with some known values and recording the corresponding changes in the intensities. A linear relationship between the known perturbation of the combined model $(\delta c)$ and know perturbation of the pose parameters $(\delta p)$ and the residual intensity values $(\delta t)$ (obtained from sum of squared difference between the intensities of the perturbed mean model and the target image) are acquired in a multivariate regression framework as,

$$
\delta c=R_{c} \delta t, \quad \delta p=R_{p} \delta t
$$

where $R_{c}$ and $R_{p}$ refer to the correlation coefficients. Given a new instance, equation (5) is used as update parameters where residual intensity value $(\delta t)$ is used to generate new pose parameters, new model parameters and hence new intensity values. The process continues in an iterative manner until the differences with the target image remains unchanged.

AAM assumes the shape space, the intensity space and hence the combined model space to be Gaussian. However, inter-patient prostate shape and intensity may vary significantly. Moreover prostate shape and intensity values vary significantly across the base, the central and the apex regions of the prostate and under such circumstances approximating with a single Gaussian mean AAM introduces segmentation inaccuracies. To address this problem we propose to use different models of the base, central and the apex regions of the prostate.

The schema for building the multiple models for the apex, central and the base regions is as follows; initially the prostate slices of the training volumes are divided into three distinct sections as the apex, the central and the base regions. For dividing the prostate we divide the number of slices of prostate (obtained from ground truth values) by 3 , the resulting quotient is used to group the slices from the top and the bottom into apex and the base groups and remaining slices of the central region are placed in one group. The objective of such grouping is to produce different mean models for each of these regions that better approximate each of these regions and improve segmentation accuracies.

Moreover for a given region (apex, central and base) multiple mean models are produced to better approximate local variability of each of the regions. The sum of squared differences of the intensities between a mean model and the target image is recorded as the fitting or registration error after the final segmentation with each of the mean model for the corresponding region (apex, central and base). The corresponding region is determined using the same method that is used to group the prostate slices during training. The segmentation result of the mean model of the corresponding region with least fitting error is considered as the optimized segmentation for that particular image. The framework of building multiple mean models for each of the region (apex, central, base) is as follows; the base region has 43 slices from 15 datasets. Initially slice 1 is selected as the reference to register slices 3 to 43 to build the mean model and test it on slice 2 and record the fitting error (sum of squared differences of the intensities between the mean model and the test image i.e. slice 2). Likewise, with the fixed reference (slice 1) we build the second mean model by registering slice 2 and slices 4-43 to test slice 3 and record the fitting error. The process is repeated for all the slices to generate 42 model fitting errors with slice 1 as the reference as shown in Fig. 3.

Consequently the reference dataset is changed from 2-43 to generate 43 model fitting error graphs (one for each slice). We have analyzed the model fitting error values and the corresponding segmentation accuracies and have observed that less fitting error translates into higher segmentation accuracies (in terms of DSC, HD etc). An empirical error value is determined from the 43 model fitting error graph (the red line $\leq 3000$ in our case) beyond which the segmentation accuracy is reduced. The reference slice that has fitting error less than this empirical value with maximum number of slices is selected, grouped together (slice 1, 4, 14, 16, 20, 27, and 41) and removed from further grouping. The process is repeated until all the slices are grouped. These groups of datasets provide individual mean models (8 mean models in our case). However, increasing the number of mean models (decreasing the fitting error threshold) improves segmentation accuracy additional computational time. Hence, the choice of optimum number of mean models is a trade off between segmentation accuracy and computational time requirement of the process. Finally, the 2D labels are rigidly registered to the 3D labels generated using probabilistic atlas to constrain pose variation and generate valid 3D shapes.

\section{Results}

We have validated the accuracy and robustness of our approach with $15 \mathrm{MR}$ public dataset with image resolution of 
Table 1. Prostate segmentation quantitative results (Time (mins/volume), HD \& MAD (mm), Spec.=Specificity, Sens.=Sensitivity, Acc.=Accuracy,

\begin{tabular}{|c|c|c|c|c|c|c|c|}
\hline Method & DSC & $\mathrm{HD}$ & MAD & Spec. & Sens. & Acc. & Time \\
\hline Merida [8] & 0.79 & 7.11 & - & - & - & & 60 \\
\hline Dowling [9] & 0.73 & - & & - & - & & 60 \\
\hline & \begin{tabular}{|l} 
\pm 0.11 \\
0.82 \\
\pm 0.05
\end{tabular} & $\begin{array}{l}10.22 \\
\pm 4.03 \mathrm{vx}\end{array}$ & & - & & & \\
\hline \begin{tabular}{|l} 
Our \\
Method
\end{tabular} & $\begin{array}{l}0.89 \\
\pm 0.11\end{array}$ & \begin{tabular}{|l|}
3.05 \\
\pm 2.25 \\
\end{tabular} & $\begin{array}{l}1.15 \\
\pm 1.05 \\
\end{array}$ & $\begin{array}{l}0.88 \\
\pm 0.08\end{array}$ & $\begin{array}{l}0.996 \\
\pm 0.006\end{array}$ & $\begin{array}{l}0.98 \\
\pm 0.07\end{array}$ & 40 \\
\hline
\end{tabular}

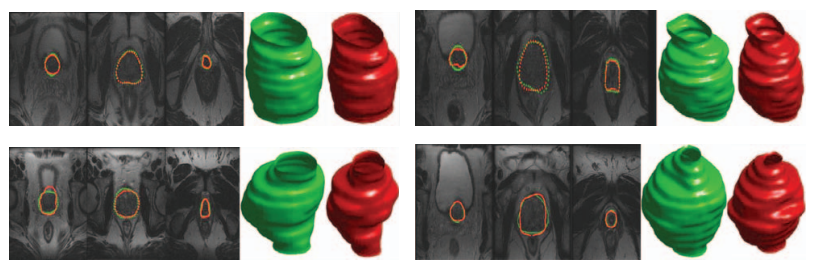

Fig. 4. Subset of segmentation results of 4 datasets. One axial slice from the apex, central and the base regions are displayed. Green contour/volume shows the ground truth and red contour/volume shows the achieved segmentation.

256x256 pixels of MICCAI prostate challenge [11] in a leaveone-out evaluation strategy. During validation the test dataset is removed and the probabilistic atlas and multiple mean models of the apex, central and the base regions are constructed with the remaining 14 datasets. To determine the region of interest for atlas based registration the center of a central slice is manually provided by the user. Such an interaction is necessary to minimize the influence of intensity heterogeneities around the prostate $[8,2]$. The probabilistic atlas produces an initial soft segmentation of the prostate. The centroid of each of the 2D slices of the prostate volume is computed from probabilistic values of the soft segmentation. All the mean models of the corresponding regions (apex, central and base) are initialized at the centroid of each of the slices to segment the prostate in that slice. The segmentation result of the mean model producing the least fitting error is selected as the final segmentation in $2 \mathrm{D}$. The $2 \mathrm{D}$ labels are rigidly registered to the $3 \mathrm{D}$ labels generated using probabilistic atlas to constrain pose variation and generate valid 3D shapes.

Our method is implemented in Matlab 7 on an Intel Quad Core Q9550 processor of $2.83 \mathrm{Ghz}$ processor speed and 8 GB RAM. We have used most of the popular prostate segmentation evaluation metrics like Dice similarity coefficient (DSC), 95\% Hausdorff distance (HD), mean absolute distance (MAD), specificity, sensitivity, and accuracy to evaluate our method. We have compared our method with the results published in MICCAI prostate challenge 2009 [8, 9] and with the work of Gao et al. [10] in Table 1. We observe that our method performs better than some of the works in literature. It is to be noted that [9] used a probabilistic atlas for their segmentation. However, our hybrid framework of probabilistic atlas and multiple SSAM improves on overlap and contour accuracies. The accuracy of our method may be at- tributed to the use of the hybrid framework of optimized 2D segmentation that incorporate local variabilities and 3D shape restriction to produce a valid prostate shape. Multiple mean models of shape and intensity priors for different regions of the prostate approximate the local variabilities better as each of these models are capable of producing new instances in a Gaussian space of shape and appearance. Also, SSAM being a region based segmentation technique performs well in the base and apex regions of the prostate for low contrast images. Qualitative results of our method are presented in Fig. 4.

\section{Conclusions}

A novel hybrid schema of atlas based segmentation and multiple statistical models of shape and intensity priors of prostate with the goal of segmenting the prostate in 3D MRI images has been proposed. Our approach is accurate, and robust to significant shape, size and contrast variations in MRI images compared to some existing work in the literature. However, the proposed method has to be validated with larger number of datasets in future. Considering that tumor was not present in the datasets used for validation large scale deformation of the prostate due to presence of tumors may reduce contour based segmentation accuracies.

\section{References}

[1] "Prostate Cancer," American Cancer Society Atlanta, GA [Online]. cancer.org, accessed on [28th June, 2011], 2011.

[2] S. Klein et al., "Automatic Segmentation of the Prostate in 3D MR Images by Atlas Matching Using Localized Mutual Information," Med. Physics, vol. 35, pp. 1407-1417, 2008.

[3] S. Martin et al., "Automated Segmentation of the Prostate in 3D MR Images Using a Probabilistic Atlas and a Spatially Constrained Deformable Model," Med. Physics, vol. 37, pp. 1579 - 1590, 2010.

[4] S. Chandra et al., "Automatic Segmentation of the Prostate in 3D Magnetic Resonance Images Using Case Specific Deformable Models," in DICTA, 2011, pp. 7-12.

[5] R. Toth et al., "A magnetic resonance spectroscopy driven initialization scheme for active shape model based prostate segmentation," Med. Imag. Anal., vol. 15, pp. 214-2225, 2011.

[6] Y. Zhu et al., "A Hybrid ASM Approach for Sparse Volumetric Data Segmentation," Pat. Recog. \& Imag. Anal., vol. 17, pp. 252-258, 2007.

[7] W. Li et al., "Learning image context for segmentation of prostate in ct-guided radiotherapy," in MICCAI, 2011, vol. 6893 of LNCS, pp. $570-578$.

[8] A. Gubern-Merida et al., "Atlas based segmentation of the prostate in MR images," wiki.namic.org/Wiki/images/d/d3/GubernMerida_Paper.pdf, accessed on [20th July, 2011], 2009.

[9] J. Dowling et al., "Automatic atlas-based segmentation of the prostate," wiki.na-mic.org/Wiki/images/f/f1/Dowling_2009 _MICCAIProstate_v2.pdf, accessed on [20th July, 2011], 2009.

[10] Y. Gao et al., "A Coupled Global Registration and Segmentation Framework with Application to Magnetic Resonance Prostate Imagery," IEEE Trans. Med. Imaging, vol. 10, pp. 17-81, 2010.

[11] MICCAI, "2009 prostate segmentation challenge MICCAI," wiki.namic.org/Wiki/index.php, accessed on [1st April, 2011].

[12] J. P. Thirion, "Image matching as a diffusion process: an analogy with Maxwells demons," Med. Imag. Anal., vol. 2, pp. 243-260, 1998.

[13] T.F. Cootes et al., "Active Appearance Models," in ECCV, 1998, pp. 484-498, Springer. 